\title{
Study on the Distributed Detection System for Digital TV Signal Based on Prognostic and Health Management
}

\author{
http://dx.doi.org/10.3991/ijoe.v9iS6.2790 \\ Rongliang Luo ${ }^{1}$, Xinyuan Zheng ${ }^{2}$ \\ ${ }^{1}$ Zhejiang University City College, Hangzhou, Zhejiang, China \\ ${ }^{2}$ Zhejiang Broadcast \& Television Technology Co., Ltd., Hangzhou, Zhejiang, China
}

\begin{abstract}
With continuous further development of cable television technology, the integrating and intelligent degree of digital TV network increases constantly. And people are paying more and more attention to issues such as the stability, reliability, maintainability, security, detectability, life cycle cost, etc. which may provide favorable conditions for the generation and development of prognostic and health management (PHM) of the network equipment. In this paper, the background knowledge of the distributed digital TV signals detection system was illustrated simply at first, and then the basic concept, function and current situation of PHM were introduced. On that basis, the system design of PHM was reflected from system structure and functional component by aiming at realizing this project. In the end, the equipment health examination module has been designed specifically from the operating principle, module composition, function realizing process and decision-making support.
\end{abstract}

Index Terms-Prognostic and Health Management, Distributed Detection System, Digital TV Signal.

\section{INTRODUCTION}

With further development of cable television technology and increase in transmission equipment, the cable television network is getting more and more complicated. The operators are confronted with higher and higher demands to effectively improve the monitoring for transmission signals for transmission equipment and broadcasting conditions for cable television, and the traditional monitoring model fails to meet the requirements. It is urgent for the radio and television to carry out automatic monitoring in the broadcasting quality, transmission quality and transmission equipment of the cable television accurately and rapidly, and to evaluate the network and equipment monitoring condition. It is in urgent need of a set of signal monitoring system, which can not only be equipped with favorable detection precision, rich detection content and flexible alarming method, but also provide monitoring software for the prediction of failure and suggestions to maintenance[1], [2].

Prognostic and Health Management (PHM) study has already become a hot spot in the development process of foreign and domestic equipment, with the PHM system of American JSF as a typical case [3]. In addition, the researches and explorations in this aspect have already been carried out in complicated systems such as the aircraft industry in China [4].

\section{INTRODUCTION TO PHM}

With the rapid development of sensor technology, embedded operating system, network technology and failure diagnosis technology, PHM has been increasingly applied into various scientific research institutions and industrial enterprises. PHM is a further development of traditional design condition monitoring capability for complicated system, which can realize the transformation from afterward remedy to prior prediction. The health condition of the system is judged and predicted by analyzing the history data and checking the current state to achieve reliable prediction for the system in the future. It can recognize and predict the occurrence of the failure with this capability, and maintain in advance, for improving the stability and reliability of the system, reducing the operating and maintenance cost, and realizing the stable operation of the system with less maintenance input [5].

Generally, PHM system will comprehensively analyze and deal with the state monitoring for electronic equipment, information of functional test, as well as the diagnosis and prediction of failure, evaluate the technical state of the current and future electronic equipment with the help of decision support technology, such as the comparison and analysis of historical data, and finally realize the health management of electronic equipment.

Data-based measure of PHM system refers to the direct deduction according to the monitoring data of the system. The health state is usually forecasted with the help of model recognition on the basis of statistics and learning, the expert system and decision support technology by employing the sensor monitoring and signals concerning the health conditions of the system or parts. On the premise of unchanged statistical characteristics of the system, the prediction and diagnosis accuracy of this method depends on the quality of the system data and the measures for prediction [6].

PHM technology has been widely used in the design and maintenance of the complicated systems for unmanned aerial vehicle, guided missile, radar, etc [7]. in China, but it is seldom applied in the civil systems, such as the cable television network. 


\section{DESIGN OF DISTRIBUTED DETECTION SYSTEM FOR DIGITAL TV SIGNAL}

With development changes in the external environment, customer demands and communication network technology, the traditional radio and television business in broadcasting cable network realizes the simulation of digital translation and digital television, on which new businesses such as broadband data, etc. and a series of value-added services have been developed. As a result, it sets a higher demand on the stability of the network operation, and it must rely on the stable and reliable network management system to meet the requirement on the reliability of carrier-class bi-directional business. In addition, owning to the dramatic increase in the quantity of equipment caused by FTTB (fiber-to-the-building), the traditional manual maintenance fails to be qualified, and it must rely on the webmaster for monitoring and management. HFC (Hybrid Fiber Coaxial) is widely spread in Chinese cable television network. The information source system of digital television includes the digital satellite signal receiving system, analog signal encoding system, signal conversion system, etc. The cable television signal will be transmitted to the optical node through cable, at which it will be converted to the radiofrequency signal and transformed to the HFC and then to the users. As the core of digital cable television system, the front-end system mainly consists of the digital television information source system, storage broadcasting system, business system, receiving system, encoding modulating system, post-back processing system, etc.

According to the actual demands, a kind of network management equipment was proposed, which is distributed online network monitoring equipment allocated on each endpoint of the cable television network to realize the multi-point monitoring for the network from the frontend of the cable television to the client. It can acquire the real-time or in-time line signals and a series of technical indexes, such as the operating condition of the equipment, cable digital television signal, cable analog television signal, cable FM-broadcast signals, etc. The index information will be sent back to the central office through IP network, present the index information and alarm information through network management system software, and evaluate the technical indexes for the equipment in monitoring region effectively through the health management system of the system software, thus to judge the operating condition and health condition of the equipment and to realize effective, visual and real-time monitoring for the signal quality and health condition of the equipment.

The overall construction of the distributed cable television signal monitoring system is shown as follows (see Fig. 1).

The distributed cable television signal monitoring system mainly consists of health management monitoring server, monitor with network management module, remote equipment (signal collector, backbone transmission equipment, optical node, amplifier, client, etc.). The data communication of the system is mainly conducted through the Ethernet.

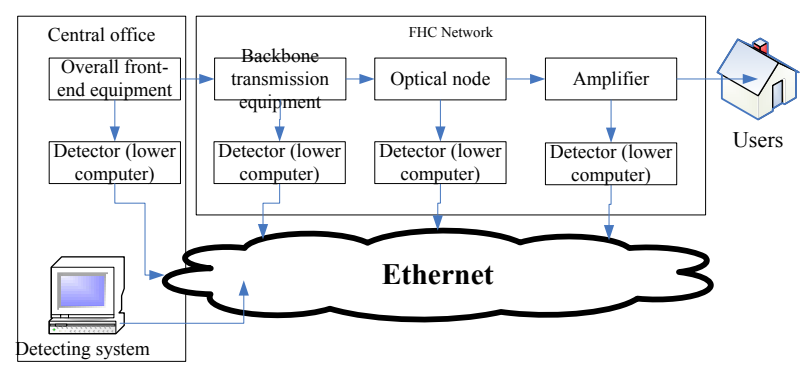

Figure 1. Overall construction figure

This design scheme mainly involves three parts, the first is the design for the monitor with network management module (lower computer), and the second is the design for the health management monitoring server (upper computer).

\section{A. Design for the monitor}

Step 1 : The establishment of the development platform.

As for the hardware, ARM7 ( $33 \mathrm{C} 44 \mathrm{~B} 0 \mathrm{X}$ ) is adopted as the CPU of the module, and 8MSDRAM, 2MNORFLASH, network, serial port and so on are adopted as the assort. As for the software, uclinux operating system, net-snmp-based management agreement (SNMP), TCP/IP-based communication protocol are adopted.

Step 2 : The functions of the network management module software.

The design of lower computer software is developed on the basis of uclinux and net-snmp.

Aiming at the requirements on the monitoring of technical index of cable television signal and the management of communication equipment, the functions of lower computer include:

The design of lower computer software is developed on the basis of uclinux and net-snmp.

A. The equipment ID and name of the network management module can be set through the server detection program, and the IP address can also be altered by the server detection program.

B. The channel table of the remote collecting module is allocated to the network management module through the server detection program, and to the remote module through the network management module.

C. Conducting real-time scanning for the remote module, and store the real-time data into the network management module. The serial communication monitoring of the network management module and remote module can be realized and reported to the network management system.

D. The network management will scan the channel table regularly, inquiry the remote data, and store the data obtained in the network management module for the acquisition needs of the network management system.

E. Channel scanning (collecting the data of each channel).

F. Scanning the real-time slope, parameters of a certain channel and frequency.

G. Reporting the alarms of the equipment. 
Other functions include the remote update function of network management module, the function of increasing GPRS, etc.

\section{ARCHITECTURE DESIGN FOR THE PHM DETECTION SYSTEM}

The distributed digital television signal PHM detection system mainly consists of the lower computer monitoring equipment and server. The lower computer includes the (overall front-end equipment, backbone transmission equipment, optical node, amplifier) sensor and related data procession unit, while the server mainly includes the device-level detection manager, system-level, data record and management module, local database, data communication agent, as shown in Fig.2. The device-level manager will implement real-time monitoring and fault detection, fault diagnosis and location for the selected monitoring equipment respectively, while the system-level manager mainly realizes functions such as real-time state detection, alarm information detection, health inspection and generation of health report, maintenance remind and guidance, generation of maintenance suggestion, etc.

Distributed digital television signal PHM detection system will send the lower computer data acquired by the sensor of corresponding equipment in the form of data package to the upper computer (server) network management module through the network, and to the data record and management module through the network management module. The data record and management module finishes the data interactions among the modules, which mainly includes the up-level application platform, external real-time status monitoring information, comprehensive security management system and local database. Meanwhile it is also equipped with the import function of the algorithm configuration information and export function of the result information. The local database stores such information as the system configuration information, equipment real-time information, equipment history information, equipment alarm information, system maintenance knowledge base, system diagnostic strategies library, etc. Of course, the upper computer detection system can also send commands to the lower computer detector, for the convenience of achieving the real-time information and working condition of the equipment. Therefore, the data transmission is bidirectional.

Aiming at the characteristics of television signal monitoring and equipment management monitoring, a detection system with fault detection and health function has been designed, and its communication agreement with the lower computer detector is based on the SNMP agreement. The network management system is based on the manager of SNMP network management agreement, namely the broker model.

The detection system mainly realizes the functional modules, including the information configuration, equipment detection, fault detection, health management, maintenance decision-making system, network management, system management, etc.

A. Information database management: it mainly realizes the configuration of channel table, alarm threshold, equipment information, etc.

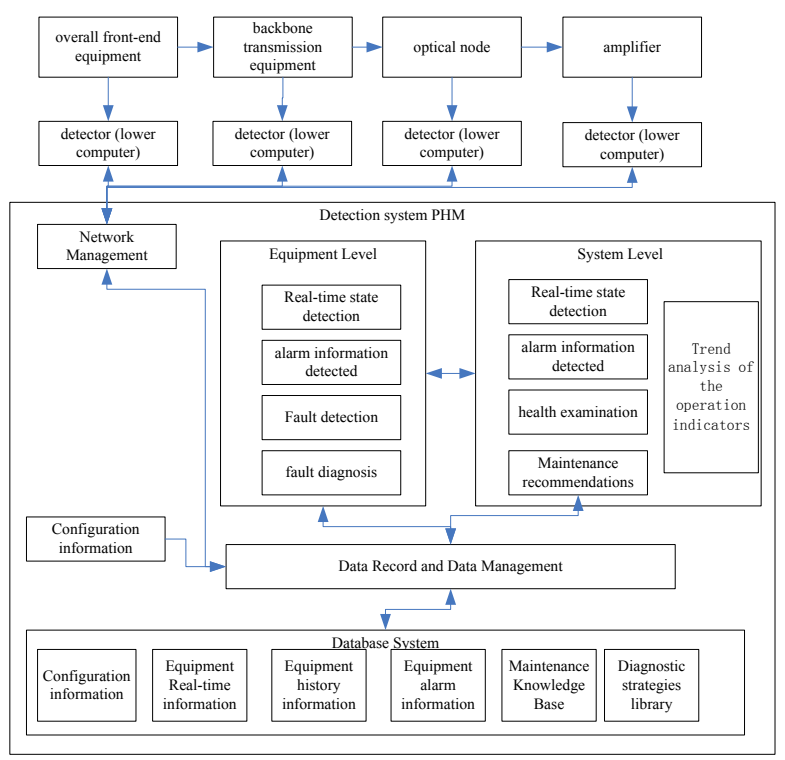

Figure 2. Architecture Diagrams

The function module is shown in Fig. 3.

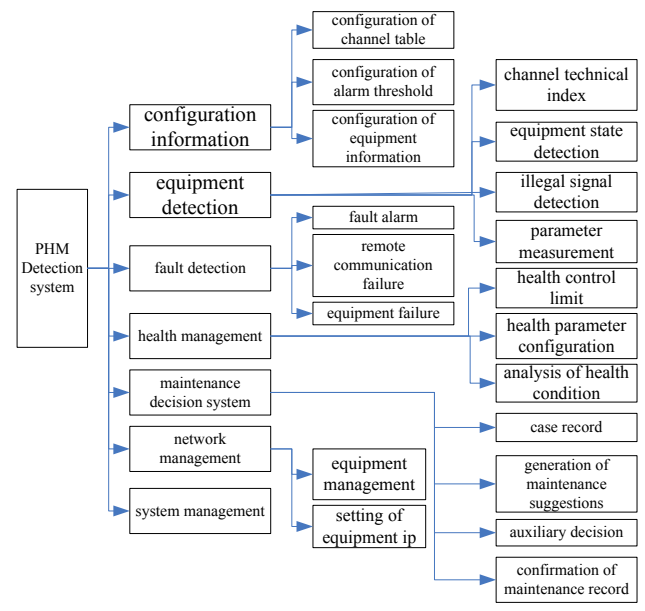

Figure 3. System Functional Diagram

B. Equipment detection: it mainly realizes the detection of channel technical index, equipment status and illegal signal, as well as the measurement of equipment parameters. The channel technical index also includes related indexes, such as the digital signal level, digital system noise level, broadband, carrier to noise ratio, bit error rate, modulation error ratio, analog signal image carrier level, sound carrier level, noise carrier level, V/A, $\mathrm{C} / \mathrm{N}$, etc.

C. Fault detection: it realizes the equipment fault alarm, the monitoring of equipment serial communication, remote communication monitoring and fault journal management.

D. Health management: it mainly finishes the settings for related characteristic parameters concerning the equipment health, the evaluation of the equipment health trend, offset state monitoring and the health state of the equipment, and the analysis and evaluation report of the health conditions.

E. Maintenance decision system: it mainly provides suggestions for the corresponding failure causes on the 
basis of decision support technology and according to the common causes and historical failure record of, and provides maintenance suggestions according to definite failure causes.

F. Network management: to increase or decrease the network management equipment within the system management, to configure the IP address information of the network management module.

G. User management: to realize multi-user management (level administrator, ordinary user), the administrator can carry out all the operations / adding and deleting user / log maintenance for all the users, while the ordinary users can only carry out the regulated operations.

Information interaction between the lower computer and detection system

The bi-directional communication of information can be realized by the lower computer and detection system. If the detection system needs to achieve the current status of some equipment, it can send commands to the lower equipment, and when the lower computer receives the command, it will analyze the command and read the relevant information of the equipment. After the lower computer achieves information, it will send the corresponding information to the detection system. The detection system will analyze the information received from the lower computer, and if the information is legal, it will process accordingly, but if it is wrong, the system will prompt error message and record in the error log. The detailed flow is shown in Fig. 4.

The information exchange format is as follows:

Information $=$ start code 1 bit + address code 2 bits + frame length 2 bits + command word 1 bit + message body $\mathrm{X}$ bits + completion code 1 bit

\section{EQUIPMENT HEALTH MANAGEMENT}

The forecast of equipment health status is realized from data analysis, which includes the historical data and data of current running state[8]. Though data analysis, the health management can be conducted for the equipment, which can help grasp and learn about the operating conditions of the equipment in time and forecast the possibility of normal operation in the future for the equipment. The method of equipment health management adopts the statistical process control as its theoretical basis, which mainly consists of equipment health inspection and equipment health evaluation [9]. In the study of PHM, the data is often divided into two types, namely the event data and status data. The event data is directly related to the failures and failure events, for instance, if the temperature of the equipment is too high, it will be deemed that the equipment will lose efficacy soon. The analysis of event data is relatively simple, which mainly adopts multivariable statistical techniques, such as main parts analysis, etc.

\section{A. Equipment status monitoring}

Normal distribution is a common statistical distribution form obeyed by the equipment health characteristic quantity. Taking the normal distribution as an example,

$$
\operatorname{MaxV}=\mu+3 \sigma \text {, NormalV }=\mu, \quad \operatorname{MinV}=\mu-3 \sigma
$$

In which $\mu$ and $\sigma$ are the mean value and standard deviation of the statistical distribution respectively. As for the normal distribution, no matter what the value of $\mu$ and

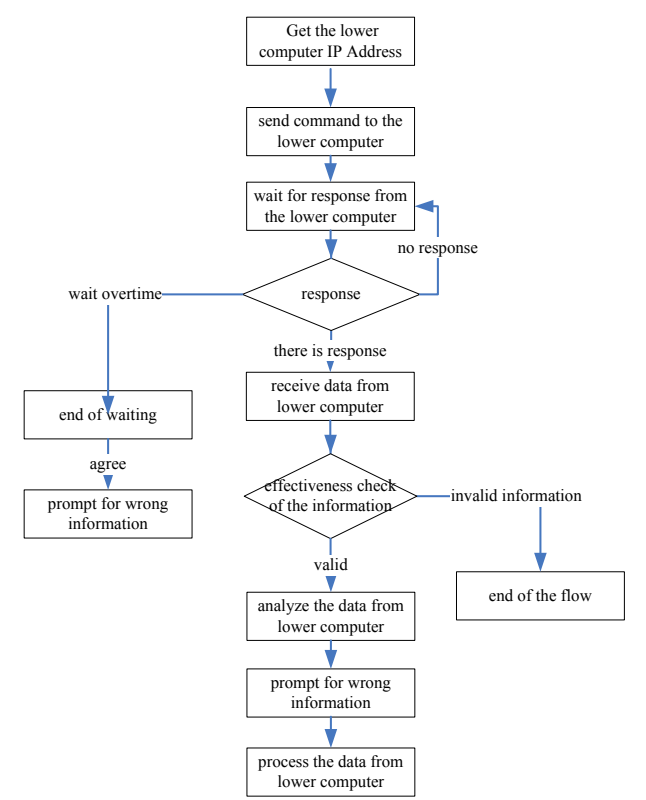

Figure 4. The flow of achieving equipment information

$\sigma$ is, the probability of being within $[\mu-3 \sigma, \mu+3 \sigma]$ is $99.73 \%$. Therefore, the health status of the equipment can be monitored accurately by analyzing the health control figure. In practice, the data distribution type of the specific object should be determined by checking the historical operating data of the equipment in advance.

\section{B. Equipment health evaluation}

The eigenvalue of the equipment under normal condition fluctuates within the acceptable scope, and the healthier the equipment is, the smaller the fluctuation is. When the equipment is unstable, the fluctuation range of the eigenvalue will increase. According to the experience, the health status of the equipment can be described through the eigenvalue fluctuations of the component. The health status of the component is represented by $\mathrm{H}$, and the health degree of this component can be represented as:

$$
\mathrm{H}=6 \sigma
$$

$\sigma$ is the standard deviation of the characteristic parameter. By calculating the value of $H$, the smaller the value of $\mathrm{H}$ is, the more stable the system will be. And the larger the value of $\mathrm{H}$ is, the more unstable the system will be.

HIdx can be employed to represent health index with the equation,

$$
\mathrm{HI}=(\operatorname{MaxV}-\mathrm{MinV}) / \mathrm{H}=(\operatorname{MaxV}-\mathrm{MinV}) / 6 \sigma
$$

The scope of HI is within $[0,1]$, the health status of different equipment can be scaled according to different practical conditions. During the transmission process of cable television signal, the significance of equipment varies. And the equipment should be set with corresponding weights according to the practical conditions.

$\mathrm{HN}$ is adopted to represent the overall health condition of the network, then

$$
\mathrm{HN}=\mathrm{a} 1 \mathrm{HI} 1+\mathrm{a} 2 \mathrm{HI} 2+\ldots+\mathrm{anHIn}
$$

In which, a1, a2, .. an are the health weights of related equipment. The larger the network health index $\mathrm{HN}$ is, the more stable the network equipment of television transmission is, and the smaller the $\mathrm{HN}$ is, the more 
unstable it will be, and network equipment with lower health index should be maintained.

\section{Realization of equipment health detection}

As for the failure information occurred, records should be kept in detain, as shown in table I. The records of the information are favorable for the conclusion and summary of problems, which can provide necessary resources for further pre-judgments.

The flow of realizing the equipment health detection function is shown in Fig. 5. The results of equipment health inspection are outputted in the form of health inspection report, which mainly includes the specific equipment, or the results of transfinite analysis, offset analysis or trend analysis of the equipment health characteristic parameters within certain time period with the reports of health condition of the equipment at current stage.

TABLE I.

FAILURE INFORMATION

\begin{tabular}{|c|c|c|c|c|}
\hline $\begin{array}{c}\text { Failure } \\
\text { Site }\end{array}$ & $\begin{array}{c}\text { Failure } \\
\text { Causes }\end{array}$ & $\begin{array}{c}\text { Possible } \\
\text { Effects }\end{array}$ & $\begin{array}{c}\text { Solutions to the } \\
\text { Failures }\end{array}$ & $\begin{array}{c}\text { Severity } \\
\text { Level }\end{array}$ \\
\hline & & & & \\
\hline
\end{tabular}

Taking the trend analysis as an example, the index information of various indexes, including the level, $\mathrm{C} / \mathrm{N}$, MER, BER, etc. of a certain monitoring point in different periods has been collected, which can be inquired through table or imaging or monitor the variation trend of a certain index automatically. Once the pre-set threshold is exceeded, it will alarm immediately. After the index trend of a certain period has been defined, the index signal of the next stage can be estimated and pre-alarmed, which can provide conditions for eliminating the failure potentials in time and reach the goal of preventing the interrupt, break and inferior transmission of the signals.

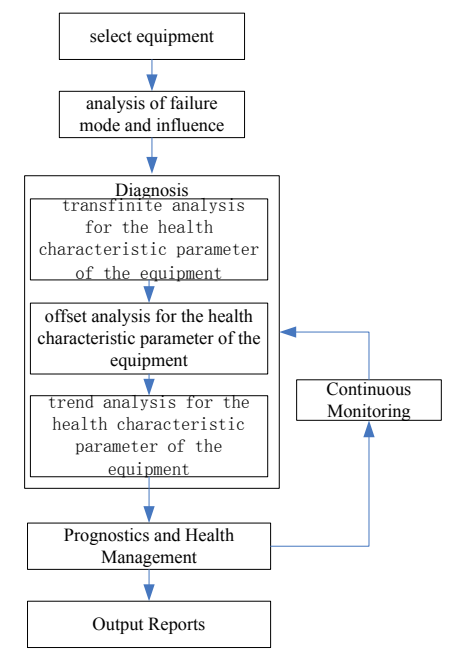

Figure 5. Flow of realizing the equipment health detection function

\section{CONCLUSIONS}

Aiming at the higher and higher demands on the stability of cable television, a distributed digital television signal detection system has been proposed, which is equipped with certain function of fault detection and health management. In this paper, it illustrates the construction and design of system structure and the functions of detector at first. Then it further illustrates the architecture of PHM detection system and gives detailed architecture diagram, so that the system is equipped with the function of primary PGM system. The information interactions between the functional modules of the PHM detection system, lower computer and detection system are illustrated with emphasis, and meanwhile, the measures for equipment health management are provided. Then the reliability of equipment will be focused on, wrong health evaluation conclusion will be studies, more automatic diagnostic method will be developed and highreliability PHM system in the whole transmission process of the digital television will be realized.

\section{REFERENCES}

[1] A. Onsy, R. Bicker, B. Shaw, "Intelligent Diagnostic Health Management of Power Transmission Systems: An Experimental Validation", International Journal of COMADEM, vol. 13, no.2, 2010, pp. 46-58.

[2] M. Singh, T. Markeset, "A Methodology for Risk-Based Inspection Planning of Oil and Gas Pipes Based On Fuzzy Logic Framework", Engineering Failure Analysis, vol. 16, no.7, 2009, pp.2098-2113. http://dx.doi.org/10.1016/j.engfailanal.2009.02.003

[3] Keller, K., Swearingen, K., Sheahan, J., "Aircraft Electrical Power Systems Prognostics and Health Management", In IEEE Aerospace Conference, 2006, pp.1380-1383.

[4] B. Jing, Z.Yang, Z. Jie, etc. "Review on validation and verification methods of PHM system", Computer Engineering and Application, vol.47 no.21, 2011, pp.23-27.

[5] L. Gangde,Y. Zhancai, "Study on Prognostics and Health Management System Modeling Technology", Measurement \& Control Technology, vol.30 No.1, 2011, pp.59-63.

[6] M. Jian, L. Chen, T. Laifa, etc. "Design of Prognostics and Health Management for Marine Main Propulsion System", Journal of Nanjing University of Aeronautics \& Astronautics, vol. 43, no. B07, 2011, pp.119-124.

[7] T.D. Batzel, D. C.Swanson, "Prognostic health management of aircraft power generators", IEEE Transactions on Aerospace and Electronic Systems, vol 45, no.2, 2009, pp.473-483. http://dx.doi.org/10.1109/TAES.2009.5089535

[8] S. Hou, Y. Li, "Short-term fault prediction based on support vector machines with parameter optimization by evolution strategy", Expert Systems with Applications, vol.36, 2009, pp.12383-12391. http://dx.doi.org/10.1016/j.eswa.2009.04.047

[9] Z. Zhou, C. Hua, H. Fan, J. Li, "Fault prediction of the nonlinear systems with uncertainty", Simulation Modelling Practice and Theory, vol.16, 2008, pp.690-703. http://dx.doi.org/10.1016/ j.simpat.2008.04.008

\section{AUTHORS}

Rongliang Luo is with the Department of Computer Science and Engineering, Zhejiang University City College, Hangzhou, Zhejiang, China (e-mail: luorl@ zucc.edu.cn).

Xinyuan Zheng is with the Department of Development, Zhejiang Broadcast \& Television Technology Co., Ltd., Hangzhou, Zhejiang, China (email: zhengxy123456@163.com).

This work is partly supported the Science Foundation of Zhejiang Province under Grand No. 2010R50009. It is an extended and modified version of a paper presented at the 2012 International Conference on Artificial Intelligence and Its Application in Industry Production (AIAIP 2012), held in Wuhan, China in December 2012. Manuscript received 04 May 2013. Published as resubmitted by the authors 26 June 2013. 